\title{
Receptor clustering affects signal transduction at the membrane level in the reaction-limited regime.
}

\author{
Bertrand R Caré C,2,3,* and Hédi A Soula $^{3,2}$ \\ ${ }^{1}$ Université de Lyon, LIRIS UMR 5205 CNRS-INSA, F-69621, Villeurbanne, France \\ ${ }^{2}$ EPI Beagle, INRIA Rhône-Alpes, F-69603, Villeurbanne, France \\ ${ }^{3}$ Université de Lyon, Inserm UMR1060, F-69621 Villeurbanne, France
}

\begin{abstract}
Many types of membrane receptors are found to be organized as clusters on the cell surface. We investigate the potential effect of such receptor clustering on the intracellular signal transduction stage. We consider a canonical pathway with a membrane receptor $(\mathrm{R})$ activating a membranebound intracellular relay protein $(\mathrm{G})$. We use Monte Carlo simulations to recreate biochemical reactions using different receptor spatial distributions and explore the dynamics of the signal transduction. Results show that activation of $\mathrm{G}$ by $\mathrm{R}$ is severely impaired by $\mathrm{R}$ clustering, leading to an apparent blunted biological effect compared to control. Paradoxically, this clustering decreases the half maximal effective dose (ED50) of the transduction stage increasing the apparent affinity. We study an example of inter-receptor interaction in order to account for possible compensatory effects of clustering and observed the parameter range in which such interactions slightly counterbalance the loss of activation of $\mathrm{G}$. The membrane receptors spatial distribution affects the internal stages of signal amplification, suggesting a functional role for membrane domains and receptor clustering independently of proximity-induced receptor-receptor interactions.
\end{abstract}

\section{INTRODUCTION}

Signalling is the process by which a external chemical signal is perceived by the cell via membrane proteins called receptors. These receptors when activated trigger a biochemical cascade inside the cell. Two important families of signalling systems are associated with two particular type of receptors: the Receptor Tyrosine Kinase (RTK) [1] and the G-protein coupled receptor (GPCR) [2, 3]. Both systems share the same common functional features. In both cases, membrane receptors, once activated by an external ligand molecule, acquire the ability to activate directly several intracellular membrane-bound proteins that relay the signal further into the cytoplasm.

Contemporary cell biology acknowledges that, among other membrane components, receptors of different signalling pathways are not homogeneously dispatched on the membrane but are oftentimes organized in clusters [4-8], possibly due to the structuration of the membrane in lipid rafts and caveolae [9-11]. According to several recent works, receptor clustering seems to play a important role in cell signalling, and influences regulatory processes such as bacteria chemical sensitivity, chemotaxis, or G-protein signalling [12-14]. Literature however does not come to a consensus regarding the effect of clustering on receptor-ligand binding dynamics and afterwards cell response. When receptors are packed together, signal-enhancing phenomena can occur, such as ligand receptor switching [15], or improved ligand-receptor and receptor-effector encounter probabilities [16-18]. Within the context of diffusion-limited reactions, Goldstein [19] argues that

* corresponding author:bertrand.care@insa-lyon.fr clustering reduces the ligand-receptor binding forward rate constant whereas Gopalakrishnan [17] proposes that clustering increases the ligand-receptor rebinding probability, and thus the cell response. However, in a previous work using individual based-model, we showed that receptor clustering induces an attenuating effect on ligand-receptor binding and leads to a decreased apparent receptor affinity $[20,21]$, in agreement with a recent study [22]. However, the step further:the effect of clustering on signal transduction at equilibrium, directly downstream of the reception stage, remains relatively unexplored.

In this work, we determine the impact of heterogeneous (and correlated) spatial receptors distributions on the dynamics of a simple canonical pathway at the transduction stage. Since such dynamics are generally studied using mean-field models articulating averaged densities of molecules using the law of mass action, it rests on the well-mixed assumption [23, 24]. This approach is not directly applicable when considering clustering which, by definition, imposes heterogeneous receptor distributions. We propose a simple individualbased computational model to explore the dynamics of a canonical signal transduction stage between a receptor $R$ and its downstream membrane-bound protein substrate G, akin to RTK and GPCR signalling systems. This computational framework allows for the simulation of transduction by heterogeneously distributed receptors, reproducing spatial distributions on the membrane observed in living cells. 


\section{MODELS}

We consider a canonical transduction pathway, described by the following reactions:

$$
\begin{gathered}
R \underset{k_{-c}}{\stackrel{k_{+\mathrm{c}}}{\rightleftharpoons}} C \\
C+G \underset{k_{-h}}{\stackrel{C k_{+h}}{\rightleftharpoons}} C+H
\end{gathered}
$$

where $G$ is a deactivated intracellular relay protein and $H$ its activated form. The activation of $\mathrm{G}$ is induced by a activated receptor $C$ whereas $R$ is its deactivated form.

In this simple model, receptor activation/deactivation is performed by an implicit ligand at constant rates : $k_{+\mathrm{c}}$ and $k_{-c}$ respectively. This means that the model only considers the phosphorylation state of the receptor intracellular subunit, regardless of possible cooperation mechanisms due to the dimeric structure of the receptor $[25,26]$. The response is evaluated by measuring the number of activated $\mathrm{G}$ molecules $(\mathrm{H})$. $\mathrm{G}$ molecules activation follows a "hit and run" scheme, meaning that a $\mathrm{G}$ molecule is activated by an activated receptor $\mathrm{C}$ to become a activated molecule $\mathrm{H}$, so the forward reaction rate depends on $c k_{+h}$. H molecules deactivation, on the other hand, is assumed to be spontaneous (phosphatases are excluded from the model), so the backward reaction rate only depends on $k_{-h}$.

\section{A. ODE for transduction dynamics at equilibrium}

The reaction set can be expressed as a system of ODE describing the evolution of the amounts of species (denominated as lowercase letters) using the law of mass action $[23,26,27]$. The equilibrium of such system yielding $c^{\star}$ of the number of activated receptor $c$ to the total receptor number $r_{0}=r+c$ is well known

$$
c^{\star}=\frac{c}{r_{0}}=\frac{k_{+\mathrm{c}}}{k_{-\mathrm{c}}+k_{+\mathrm{c}}}=\frac{\rho}{1+\rho}
$$

with $\rho=\frac{k_{+\mathrm{c}}}{k_{-\mathrm{c}}}$.

The ratio $\rho$ thus represents the implicit ligand stimulus applied to the system.

Activation of $\mathrm{G}$ molecules occurs at a rate proportional to $k_{+\mathrm{h}} c$, and deactivation at a constant rate $k_{\text {-h }}$. The fraction of activated $h^{\star}$ (ratio of $h$ to $g_{0}=g+h$ ) is at equilibrium

$$
h^{\star}=\frac{h}{g_{0}}=\frac{r_{0}}{r_{0}+\left(1+\frac{1}{\rho}\right) \kappa}
$$

with $\kappa=\frac{k_{\text {-h }}}{k_{+\mathrm{h}}}$.

Both Eq. (3) and (4) exhibit several measurable values that relates to dose-responses curves. First let's rewrite Eq. 4 as

$$
h^{\star}=\frac{\frac{r_{0}}{r_{0}+\kappa} \rho}{\rho+\frac{\kappa}{\kappa+r_{0}}}
$$

which expresses another Michaelian-like equation but with a new saturation plateau $h_{\max }^{\star}-$ whenever $\rho \mapsto \infty-$ and the half maximal efficient ligand stimulation (often referred to as the ED50) which is in our case the ratio $\rho_{50}$ that generates half of the maximal $\mathrm{G}$ activation namely $h_{\max }^{\star} / 2$. Assuming we can measure both values from dose-responses, we can extract an equation for the reaction affinity $\kappa$ derived from either the maximal $\mathrm{G}$ activation

$$
\kappa_{\mathrm{hmax}}=r_{0}\left(1 / h_{\max }^{*}-1\right)
$$

This relation is obtained from Eq. 5 by letting $\rho \mapsto \infty$ and rearranging to obtain $\kappa$ from $h_{\max }^{\star}$. The other options is by measuring $\rho_{50}$ the value of $\rho$ that yields $h_{\max }^{\star} / 2$ that is

$$
\rho_{50}=\frac{\kappa}{\kappa+r_{0}}
$$

and with rearranging yields

$$
\kappa_{\rho 50}=\frac{\rho_{50} r_{0}}{1-\rho_{50}}
$$

Note that both $\kappa$ should be equal provided that the reaction dynamics obeys Eq. 4. Please also note that, in both cases, the greater the $\kappa$, the more ligand stimulation needed to generate a given response, so the parameter $\kappa$ is inversely proportional to the transduction reaction affinity.

Since ODE model is non-spatial it does not take into account receptor clustering. However, we will compare the theoretical dynamics of a well mixed transduction pathway with the ones obtained in simulation for heterogeneous receptors distributions which should coincide.

\section{B. Monte Carlo microscopic lattice model}

We developed a computational model that recreates the canonical transduction pathway described above, using a classical Monte Carlo microscopic lattice framework. The membrane is modelled as a $2 \mathrm{D}$ square lattice with periodic boundary conditions. We will assume that receptors are fixed at specific discrete locations on the lattice and do not impair the diffusion of $\mathrm{G}$ molecules. Receptors are set at the start of the simulation either uniformly (homogeneous distribution), or arranged in hexagonal clusters located randomly on the lattice (clustering). Additionally, crowding is ignored - several G molecules can reside on the same lattice at any given time step, but not receptors. These assumptions are imposed in order 
to restrict the study of receptor clustering to the effect of spatial correlation only, and avoid the interference of steric hindrance aspects such as macromolecular crowding and fractal diffusion $[28,29]$ in the observed effect of clustering.

The diffusion is a discrete-time random walk on the lattice. Within the Monte Carlo stochastic simulation framework, Each $\mathrm{G}$ or $\mathrm{H}$ molecule has a probability $p_{D}$ to jump to each of the four adjacent lattice node, and conversely a probability $1-4 p_{D}$ to stay on its current lattice cell, at any time step. We set $p_{D}=1 / 4$, which gives a simulated macroscopic diffusion coefficient $D=\frac{l^{2}}{4 \Delta t}$ depending on the lattice spacing $l$ and time step $\Delta t$.

A bimolecular reaction defined by a macroscopic rate $k$ expressed in $\mathrm{M}^{-1} \mathrm{~S}^{-1}$ ( such as $C+G \stackrel{k_{+h}}{\longrightarrow} C+H$ ) must be converted into a dimensionless probability $p$ of reaction whenever two potentially interacting molecules are on the same lattice cell during a given time step. The scaling from $k$ to $p$ is defined following the expression [30] :

$$
p=\frac{k \Delta t}{\mathcal{A} V_{n}}
$$

where $\mathcal{A}$ is Avogadro's number, $V_{n}$ is the volume of a lattice node. The lattice spacing $l$ and the time step $\Delta t$ therefore define the range of macroscopic reaction rates that can be simulated, so that $p \leq 1$. For unimolecular reactions (such as $H \stackrel{k_{-h}}{\longrightarrow} G$ ), the macroscopic reaction rate $k$ expressed in $\mathrm{s}^{-1}$ is converted to a dimensionless probability $p$ following the expression $p=k \Delta t$. Unimolecular reaction such as spontaneous receptor activation / deactivation, and $\mathrm{H}$ molecule deactivation, occur independently of the molecule position. Notably, although receptors are correlated in space, their activation is a stochastic process independent of their position or their neighborhood.

A simulation using an homogeneous receptor repartition should yield doses-responses curves following Eq. 3 and Eq. 4. The effects of clustering can then be measured by positioning adequately receptors and relaunch the simulations with identical parameters.

\section{RESULTS}

Parameters were defined considering a typical eukaryotic cell of radius $10 \mu \mathrm{m}\left(\sim 1.210^{3} \mu \mathrm{m}^{2}\right)$ with $10^{4}$ receptors (yielding a concentration of 8 receptors per $\mu \mathrm{m}^{2}$ ) [23], and 20 times more intracellular signalling relay proteins, consistent with typical signalling systems such as the insulin pathway [26] or the $\beta$-adrenergic pathway [31]. Taking a smaller membrane patch of $800 \times 8002$ D-lattice, with a spacing $l=10 \mathrm{~nm}$ close to the typical membrane receptor diameter [32, 33], which gives $r_{0} \sim 512$ receptors - converted down to $r_{0}=500$ for simplicity - and $g_{0}=10^{4} \mathrm{G}$ molecules.
With $\Delta t=10^{-6} \mathrm{~S}$ and $l=10 \mathrm{~nm}$, we obtain a diffusion coefficient $D=2.5 \times 10^{-7} \mathrm{~cm}^{2} \cdot \mathrm{s}^{-1}$ consistent with the fastest diffusion regime for GPI-anchored proteins on the membrane $[34,35]$.

Receptor activation and deactivation occur for each individual receptor at each time step with the respective probabilities $p_{+\mathrm{c}}$ and $p_{\text {-c }}$. Dose-responses curves were obtained by simulating different levels of ligand stimulation $\rho=p_{+\mathrm{c}} / p_{\text {-c }}$, reproduced by varying the parameter $p_{\text {-c }}$ and using a fixed value for $p_{+\mathrm{c}}=10^{-2}$. The higher the value of the parameter $\rho$, the higher the average number of activated receptors at equilibrium. These parameters were used for each simulation used in this work. The opposite approach for varying $\rho$, that is changing $p_{+\mathrm{c}}$ with a fixed value for $p_{\text {-c }}$, was also tested and led to identical results (data not shown).

The rate $k_{+\mathrm{h}}$ for the reaction $C+G \rightarrow C+H$ was defined so activation of $\mathrm{G}$ is in the reaction-limited regime to limit the effect of diffusion on the reaction rates. The transport rate $k_{t}$ of a $\mathrm{G}$ molecule to a $\mathrm{C}$ molecule was calculated as $k_{t}=\frac{2 \pi D}{\ln (b / s)}[23,36]$, where $\mathrm{b}$ is half the mean distance between receptors molecules, and $\mathrm{s}$ is the minimal reaction distance, equal to $l$ in our model. The regime of the reaction can be determined by comparing the transport rate $k_{+}$to the forward reaction rate $k_{+\mathrm{h}}$ : in order to be in the reaction limited regime, one has the condition $r_{0} k_{+\mathrm{h}} / k_{t} \geq 1$. For our lattice spacing $l$ and time step $\Delta t$, this gives the condition $k_{+\mathrm{h}} \geq 10^{7} \mathrm{M}^{-1} \cdot \mathrm{s}^{-1}$ for the forward reaction rate. Therefore we set the activation reaction probability $p_{+\mathrm{h}}=0.1$ (per $\Delta t$ ) for a $\mathrm{G}$ molecule located on the same lattice node as a $\mathrm{C}$ molecule, which gives $k_{+\mathrm{h}}=6.10^{7} \mathrm{M}^{-1} \cdot \mathrm{s}^{-1}$. We reproduced different $\mathrm{G}$ activation affinities $\kappa=k_{-\mathrm{h}} / k_{+\mathrm{h}}$ by fixing $k_{+\mathrm{h}}$ and varying $k_{\text {-h }}$ between $10^{1} \mathrm{~s}^{-1} \mathrm{~s}$ and $10^{3} \mathrm{~s}^{-1}$, which translates into probabilities of deactivation per time step $p_{-c}$ between $10^{-3}$ and $10^{-5}$.

All receptors were initialized as deactivated. Clustering is achieved by assigning fixed position for the receptors on the grid. First a number $n$ of receptors per cluster is defined, and the number of clusters is derived. Then the center of all clusters are positioned randomly, forbidding overlap. When the center of the cluster is positioned, all receptors of this cluster are set in a hexagonal tiling, spiralling around the center, which imposes an approximately disc-like shape although the lattice is square. Each cluster is randomly rotated on itself so no privileged orientation exists for non-symmetrical clusters. Note that when the cluster size is 1 - no clusters - receptors are positioned randomly on the membrane. On the other hand, a cluster size of 500 is one disc whose center is set randomly. Finally, initial positions for G molecules are set randomly, each $G$ in a initially deactivated state. Simulations were performed for a minimum of $0.1 \mathrm{sec}-$ ond of real time. For dose-response curves, we checked that steady-state was reached by veryfying the detailed 

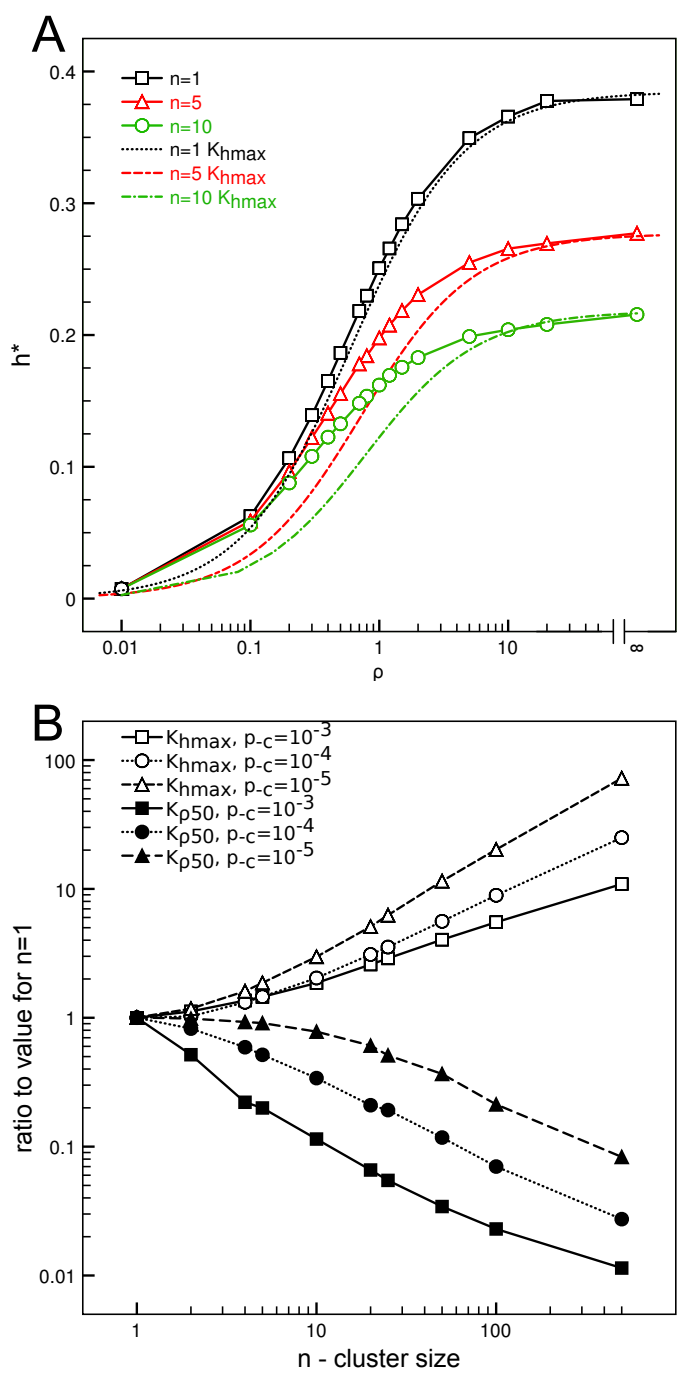

FIG. 1. A. Dose-response curves obtained in simulation for $\mathrm{n}=1$ (no cluster, open squares), $\mathrm{n}=5$ (clusters of 5 receptors, open triangles) and $\mathrm{n}=10$ (open circles), all simulation parameters remaining equal $\left(p_{+\mathrm{h}}=0.1, p_{-\mathrm{h}}=10^{-4}\right)$. Increasing levels of receptor stimulation are achieved by tuning the value of $\rho=p_{+\mathrm{c}} / p_{\text {-c }}$, with fixed $p_{+\mathrm{c}}=0.01$ and $p_{\text {-c }}$ varying. $\rho \mapsto \infty$ was obtained by setting $p_{+\mathrm{c}}=1.0$ and $p_{-\mathrm{c}}=0$. Data points were obtain by averaging $h$ at equilibrium for the last 100 time steps of 10 simulation runs. Theoretical curves (dashed lines) were obtained using Eq. 4 and $\kappa=\kappa_{\text {hmax }}$ estimated from the saturation plateau (Eq. 6). B. Values of $\kappa$ computed from the saturation plateau $\left(\kappa_{\mathrm{hmax}}\right)$ or from the half maximal efficient dose $\left(\kappa_{\rho 50}\right.$ as in Eq. 8$)$. For each curve, values were normalized on the estimate obtain for $n=1$.

balance equilibrium of the number of molecules in each state. 16 runs were performed with different pseudorandom number generator seeds for each parameter set.

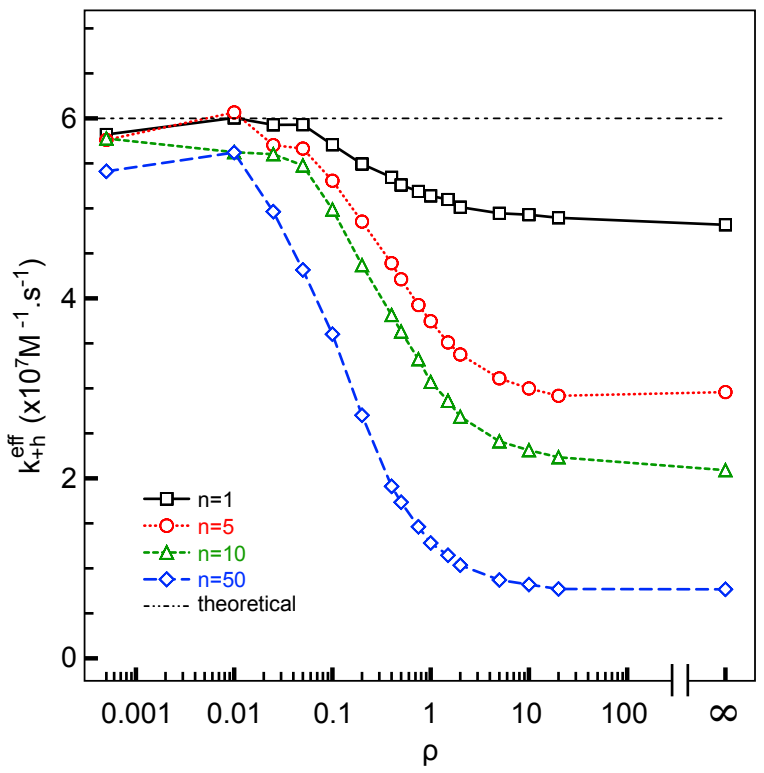

FIG. 2. Effective forward reaction rate $k_{+\mathrm{h}}^{\text {eff }}$ estimated at equilibrium for different stimulation levels $\rho$, and for different cluster sizes $\mathrm{n}$. $k_{+\mathrm{h}}^{\text {eff }}$ was obtained by measuring the number of $\mathrm{G}$ molecule activation events per time step, normalized by $h, c$, and expressed in $M^{-1} s-1$ according to lattice spacing $l$ and $\Delta t$. The irregularly dashed-line correspond to the reactionlimited theoretical rate $k_{+\mathrm{h}}$, expressed in $\mathrm{M}^{-1} \mathrm{~s}-1$ according to simulation parameters $p_{+\mathrm{h}}, l$ and $\Delta t$.

\section{A. Clustering decreases the activation of $\mathrm{G}$.}

For several values of $\rho$ ranging from 0.01 to 20 and for two cluster size $(n=1, n=5$ and $n=10-$ see Fig. $1 \mathrm{~A}$ ) the equilibrium fraction of activated $\mathrm{G}-h^{\star}-$ was retrieved. At equal receptor stimulation, clustering induces a dramatic decrease in $\mathrm{G}$ activation at equilibrium, for all values of $\rho$. Even when fully and constantly activated, receptors distributed in clusters of 5 and 10 activated less $\mathrm{G}$ than when randomly spread and separated.

This decreasing response effect is more pronounced the higher the clustering. As a way of quantifying this effect, we estimated the apparent affinity of the reaction $C+G \rightarrow C+H$ by calculating the parameter $\kappa$ using the two different methods describe in Models. We first obtain the information of the saturation plateau $h_{\max }^{\star}$ by taking the equilibrium values for very high $\rho$, averging the value of $h$ for the last 100 time steps of 10 simulation runs at equilibrium. We retrieved the half maximal efficient ligand stimulation $\rho_{50}$ by non-linear fitting of the equation $h=h_{\max } \rho /\left(\rho_{50}+\rho\right)$ on doses-responses curves that were obtained for 10 simulations runs. Using Eq. 6 we derive $\kappa_{\text {hmax }}$ and using Eq. 8 we get $\kappa_{\rho 50}$ as in to Fig. 1 B.

Surprisingly, although both estimation methods were derived from Eq. 4, they exhibit an opposite behavior with increased clustering : whereas $\kappa_{\text {hmax }}$ increases up 
to 2 orders of magnitude, $\kappa_{\rho 50}$ decreases down to 2 orders of magnitude. This phenomenon can be seen on doses-responses curves : they have a lower saturation plateau, but it is reached sooner in terms of ligand stimulation. Theoretical doses-responses curves using Eq. 4 and $\kappa=\kappa_{\text {hmax }}$ are compared to simulated dosesresponses in Fig. $1 \mathrm{~A}$ to illustrate this phenomenon. The deterring effect of clustering is somewhat mitigated by an apparent increase in sensitivity (less ligand stimulation is required) compared to its respective maximal response (which is lower than for the homogeneous case anyway). In other words the overall response is blunted whereas its sensitivity is increased. In order to confirm this effect, we measured the effective forward reaction rate $k_{+\mathrm{h}}^{\mathrm{eff}}$ for $\mathrm{G}$ activation, by tracking the number of reaction events per time step. Fig. 2 shows $k_{+h}^{\text {eff }}$ versus different levels of stimulation $\rho$, for different cluster sizes. When clustering is introduced, the effective forward reaction rates does not change compared to homogeneous distributions for low stimulation levels. But when a stronger stimulation is applied to the system, the effective rate dramatically decreases. In the homogeneous case, the effective rate stays relatively constant with respect to stimulation. This shows that the response is more affected by clustering at high levels of stimulation, where $k_{+h}^{\text {eff }}$ is strongly decreased, than at low stimulation levels, whereas in the equations predicting the dose-response curve $k_{+h}^{\text {eff }}$ is expected to be constant.

This impact of receptor clustering can be further assessed by inspecting waiting times between activation events of the same $\mathrm{G}$ molecule. In previous works with ligand-receptor binding, it was shown that the rebinding time decreased with clustering while the time before first binding increased [20-22]. Due to the nature of the problem - most ligand bound then got back to the medium - the time before first binding was a dominating feature. As such, both effects countered themselves but the depleting effect of clustering on the time before first binding was eventually stronger. In this case, the distribution of the times between two activation events are displayed on Fig. $3 \mathrm{~A}$ for various cluster sizes. Clustering induces a redistribution of the times between consecutive activations events of the same $G$ molecule. In the highly clustered case, most reactivation events occur on a very short time scale. The waiting times whose distribution is plotted on Fig. $3 \mathrm{~A}$ include the time spent waiting for deactivation. We detangle this combined waiting time by measuring the time between deactivation and reactivation of the same $\mathrm{G}$ molecule. Fig. $3 \mathrm{~B}$ shows the empirical distributions of such times for different cluster sizes. These events were also redistributed towards short times at the expense of long times, showing that randomization of molecule position by diffusing while remaining activated does not overcome the attenuating effect of clustering. We did not observe a modification of the mean waiting time in either case, for any cluster size, only a redistribution. At times near the average time
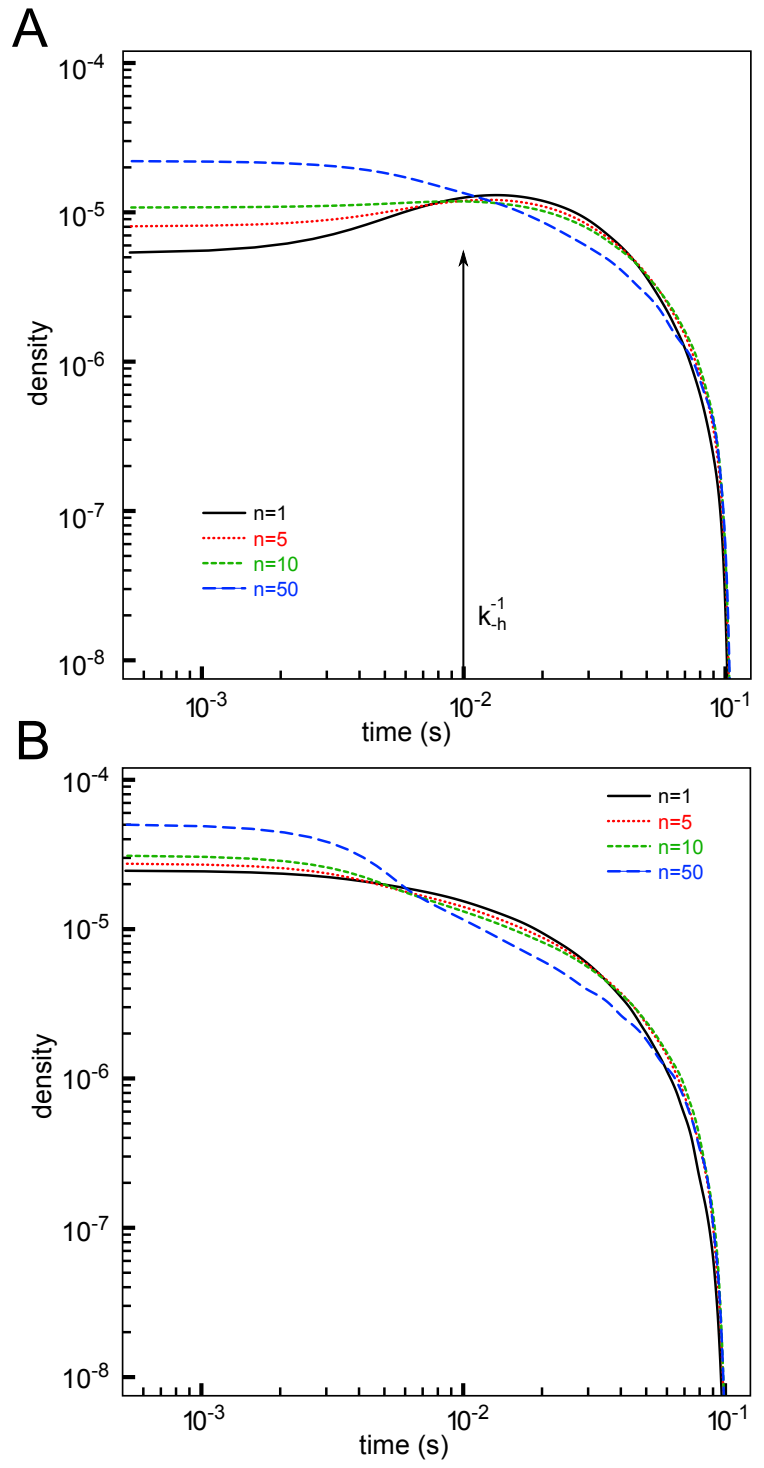

FIG. 3. A. Empirical densities of times between consecutive activation events of the same $\mathrm{G}$ molecule, using the same set of parameters $p_{+\mathrm{c}}=p_{\text {-c }}=0.01, p_{+\mathrm{h}}=0.1, p_{-\mathrm{h}}=10^{-4}$, for various cluster sizes $n$. The mean activated time for $G$ molecules is $1 / k_{-\mathrm{h}}=10^{-2} \mathrm{~s}$. B. Empirical densities of times between deactivation and reactivation events of the same $G$ molecule, using the same set of parameters, for various cluster sizes $n$.

before deactivation and larger, less reactivation events occurred.This explains the impairment of the response provoked by clustering: in this model, an activated particle can cover a lot of membrane area before deactivation. Essentially, it means that after being activated, the position where a molecule can be reactivated is anywhere on the membrane, and decorrelated from the starting position. This strongly favors the non-clustered case in terms of signal amplitude, but the clustering case in terms of response sharpness. 

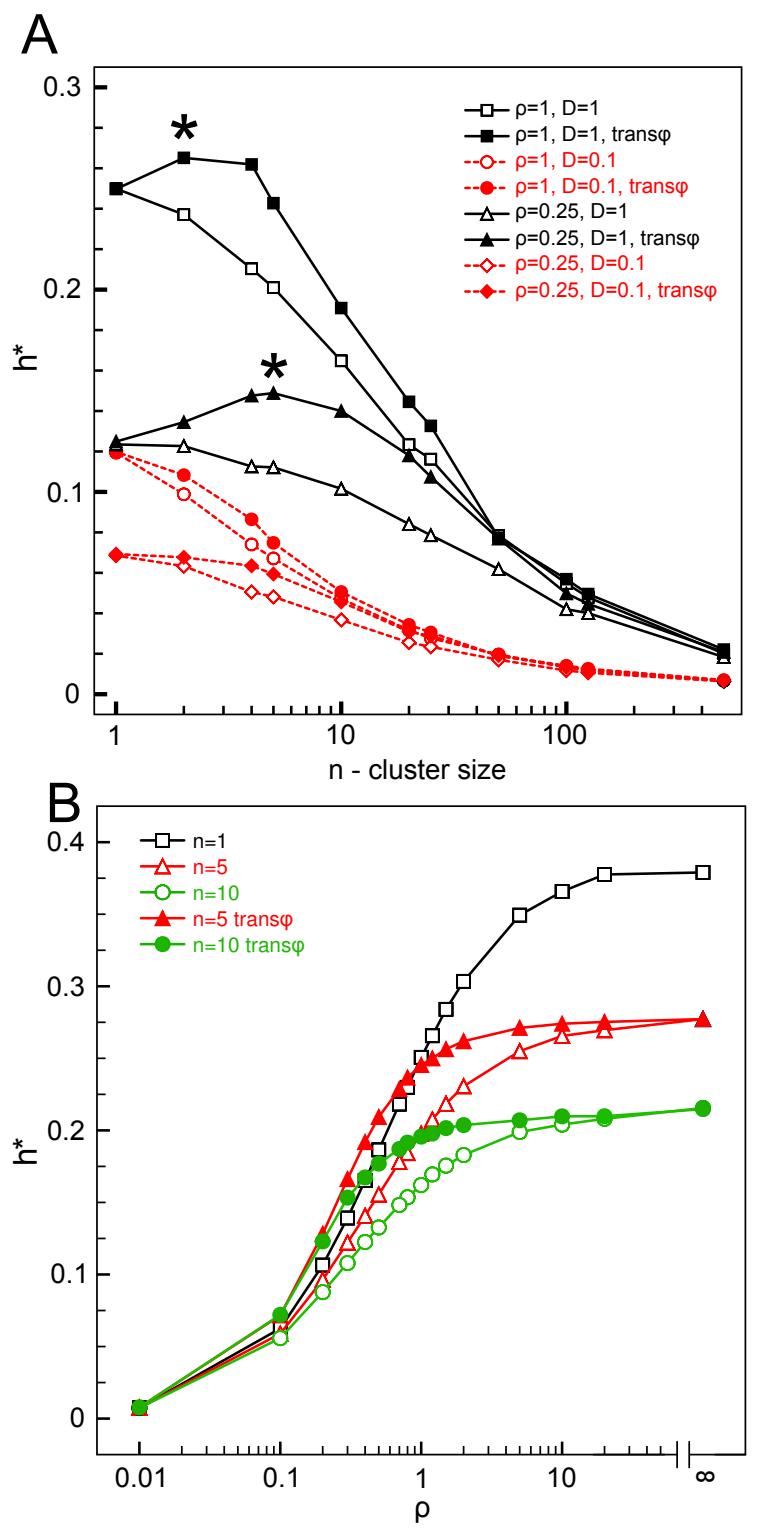

FIG. 4. A. Fraction of activated $\mathrm{G}$ at equilibrium $h^{\star}$ for $\rho=1$ (squares) and $\rho=0.25$ (triangles) versus various cluster sizes and for different diffusion coefficients D - transactivation is disabled (open symbols) or enabled (closed symbols). Optimal cluster sizes were marked with *. Parameters : $p_{\phi}=p_{+\mathrm{c}}=0.01$ and $p_{-\mathrm{h}}=10^{-4}, D=1.0$ (solid lines) and $D=0.1$ (dashed lines). B. Dose-responses curves from simulation for $n=1$ (squares), 5 (triangles) and 10 (circles). Ligand stimulation was reproduced $\mathrm{G}$ activation probabilities were $p_{+\mathrm{h}}=0.1$ and $p_{\text {-h }}=10^{-4}$, diffusion coefficient $D=1.0$. Open symbols are for simulations without transactivation whereas closed symbols are when transactivation is enabled with $p_{\phi}=p_{+\mathrm{c}}=0.01$.

\section{B. Impact of transactivation as a compensatory mechanism}

The activation of an individual receptor was until there decorrelated in time and space from the activation

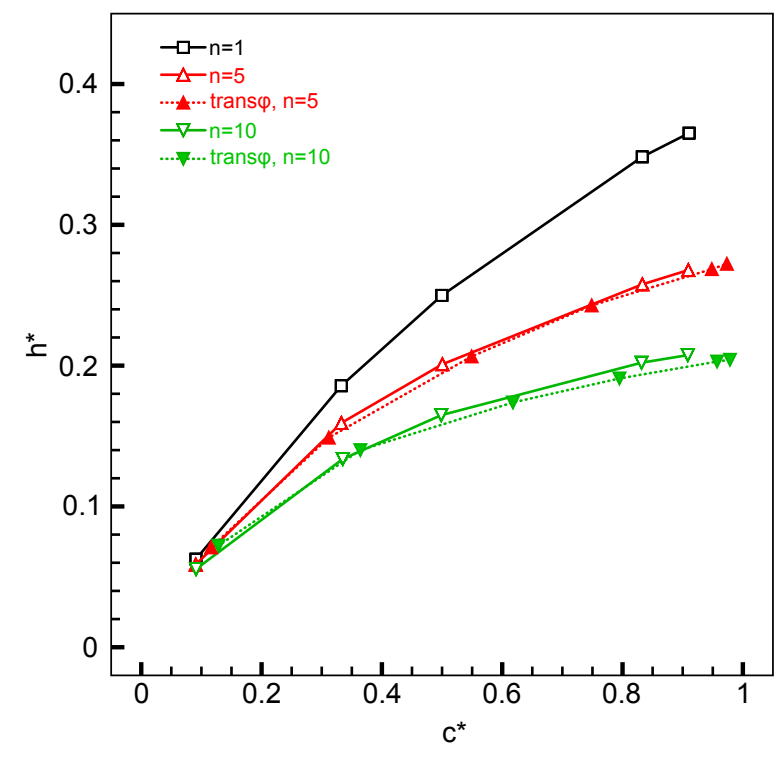

FIG. 5. Normalized number of activated G molecules $h^{*}$ versus normalized number of activated receptors $c^{*}$, for different cluster sizes $\mathrm{n}$, and diffusion coefficient $\mathrm{D}=1.0$ or $\mathrm{D}=$ 0.1. Filled symbols are for transactivation with probability $p_{\phi}=p_{+\mathrm{c}}=0.01$, open symbols are for regular reaction system without transactivation. At equal fraction of activated receptor $c^{*}$, the fraction of activated $\mathrm{G}$ molecules is the same with or without transactivation for all cluster sizes tested.

of other receptors. We explored the effect of transactivation [37-40] as an example of receptor-receptor interaction that introduces a spatio-temporal correlation in receptor activation. Within this mechanism, an activated receptor intracellular domain has the ability to activate another receptor intracellular domain located in its vicinity. It can be introduced naturally in the computational model by setting a probability $p_{\phi}$ to activate a receptor located less than 2 lattice nodes away from an activated receptor. With the hexagonal tiling used for clusters, this amounts to only the 6 closer receptors for the first step propagation.

In simulations, as expected, increasing cluster size leads to an increasing activation of $\mathrm{R}$, since larger clusters make transactivation more efficient. Transactivation can propagate itself to a larger number of receptors. In the homogeneous case, the number of activated receptors remained globally unchanged. Fig. 4 A shows the impact of transactivation on $\mathrm{G}$ activation as a function of the cluster size, for two distinct ligand stimulations levels $\rho$. Compared to the unclustered case, the addition of transactivation slightly compensates the deterring effect of clustering for small to intermediate cluster sizes. Thus, for a given stimulation, there is an optimal cluster size that maximizes $\mathrm{G}$ activation. However, since the maximal receptor activation (at maximum stimulation) remains unchanged via transactivation, for high ligand stimulation clustering still strongly impairs the overall G activation (see Fig. 4 B). However, the sensitivity (the 
stimulation needed to elicit half of the relative maximal response) is increased via transactivation, reshaping doses-responses curves towards a more "on/off" profile. One may notice that as more receptors are activated at the same value of $\rho$ when transactivation is enabled, the dose-response relationship should be estimated at equal receptor activation, so we also measured dose-response curves as the fraction of activated $\mathrm{G}$ molecules $h^{*}$ versus the fraction of activated receptors $c^{*}$, with and without transactivation. Fig. 5 shows that at equal receptor activation, transactivation does not increase the response. This means that the compensatory effect of transactivation is only due to a greater number of activated receptors at equal stimulation, and is not due to receptor clustering. Our results indicated tha clustering induces the redistribution of reactivations events towards short times at the expense of long times. The introduction of transactivation showed that having clusters of receptors activated together in a correlated manner does not increase the response, only the number of activated receptors matters. Fig. 5 also shows that diffusion does not qualitatively change the effect of clustering, with or without transactivation. The system became diffusionlimited, and the effect of clustering scales with the loss of response due to the diffusion limit.

\section{DISCUSSION}

We developed a simple individual-based spatiallyresolved computational model in which heterogeneous receptor distributions could be reproduced. The simulation of a canonical signalling pathway showed how the heterogeneous distributions of signalling proteins observed in cells can have an effect on the dynamics of transduction. A divergence with classical ODE models was observed without invoking complex protein-protein interaction mechanisms, but simply by changing the spatial distribution of receptors.

The activation of a membrane intracellular signalling protein by receptors in clusters is dramatically decreased whereas the number of available activated receptors is the same. Although the maximal amplitude of the signal was reduced, clustering decreased the half maximal efficient ligand stimulation, producing steeper doses-responses curves. This differential effect on the response appeared in the effective forward reaction rate, which becomes stimulation-dependent when clustering is induced. Since spatial clustering is available in our individual-base model framework, spatial inter-receptor interactions such as transactivation can be naturally introduced. Such correlating compensatory mechanism in receptor activation did not recover the maximal response, but accentuated the steepness of doses-responses curves. The deterring effect of clustering was partially explained by a redistribution of the waiting times between consecutive activations of the same molecule, which favored short-time reactivation at the expense of mean-time reactivation.

To investigate the effect of clustering in the least favorable conditions, this work was done in the context of the reaction-limited regime. However membrane-bound intracellular proteins also exhibit a slow diffusion regime [35]. Our previous results suggest that slower diffusion regimes would reinforce the effect of clustering [20]. The model assumes that receptor diffusion is extremely slow compared to membrane-bound intracellular protein diffusion [41], so receptors are immobile throughout simulation. Allowing receptor mobility would require the use of a dynamical clustering mechanism, possibly ligand-dependent or diffusion-dependent [42-44]

The observed transduction dynamics suggests that clustering could be a simple, effective way of modulating the response of a signalling pathway, as observed in [45]. By adapting the distribution of receptors, the dynamic range and the sensitivity can be adjusted. Our results also support the possibility that clustering is a pathway-tuning mechanism per se without invoking complex protein-protein interactions such as oligomerization, crosstalk or trafficking. The qualitative and quantitative divergences between the classical ODE system and the simulated doses-responses curves also indicates that accurate estimations of reaction rates in vivo could not be achieved without taking into account the heterogeneous spatial distributions of reactants, especially for signalling systems.

\section{ACKNOWLEDGMENTS}

This work was financially supported by la Région Rhône-Alpes. We gratefully acknowledge support from the CNRS/IN2P3 Computing Center (Lyon/Villeurbanne - France), for providing a significant amount of the computing resources needed for this work.
[1] M. A. Lemmon and J. Schlessinger, Cell, 141, 1117 (2010), ISSN 1097-4172, PMID: 20602996.

[2] W. M. Oldham and H. E. Hamm, Nature reviews. Molecular cell biology, 9, 60 (2008), ISSN 1471-0080, PMID: 18043707.

[3] R. Nygaard, T. M. Frimurer, B. Holst, M. M. Rosenkilde, and T. W. Schwartz, Trends in pharmacological sciences,
30, 249 (2009), ISSN 0165-6147, PMID: 19375807.

[4] M. Karlsson, H. Thorn, A. Danielsson, K. G. Stenkula, A. Öst, J. Gustavsson, F. H. Nystrom, and P. Strålfors, European Journal of Biochemistry, 271, 2471 (2004), ISSN 1432-1033.

[5] S. J. Plowman, C. Muncke, R. G. Parton, and J. F. Hancock, Proceedings of the National Academy of Sciences of 
the United States of America, 102, 15500 (2005), ISSN 0027-8424, PMID: 16223883.

[6] S. Lee, J. Mandic, and K. J. Van Vliet, Proceedings of the National Academy of Sciences of the United States of America, 104, 9609 (2007), ISSN 0027-8424, PMID: 17535923 PMCID: 1887608.

[7] M. Scarselli, P. Annibale, and A. Radenovic, The Journal of Biological Chemistry (2012), ISSN 1083-351X, doi: 10.1074/jbc.M111.329912, PMID: 22442147.

[8] A. N. Bader, E. G. Hofman, J. Voortman, P. M. P. v. B. en Henegouwen, and H. C. Gerritsen, Biophysical Journal, 97, 2613 (2009), ISSN 1542-0086, PMID: 19883605.

[9] K. Simons and D. Toomre, Nature Reviews. Molecular Cell Biology, 1, 31 (2000), ISSN 1471-0072, PMID: 11413487.

[10] D. Lingwood and K. Simons, Science, 327, 46 (2010), ISSN 0036-8075, 1095-9203.

[11] V. L. Reeves, C. M. Thomas, and E. J. Smart, Advances in Experimental Medicine and Biology, 729, 3 (2012), ISSN 0065-2598, PMID: 22411310.

[12] D. Bray, Annual Review of Biophysics and Biomolecular Structure, 27, 59 (1998), ISSN 1056-8700, PMID: 9646862.

[13] B. A. Mello, L. Shaw, and Y. Tu, Biophysical journal, 87, 1578-1595 (2004), ISSN 0006-3495.

[14] M. Fallahi-Sichani and J. J. Linderman, PLoS ONE, 4, e6604 (2009), ISSN 1932-6203.

[15] P. A. Mahama and J. J. Linderman, Biophysical Journal, 67, 1345 (1994), ISSN 0006-3495, PMID: 7811949.

[16] L. D. Shea and J. J. Linderman, Journal of Theoretical Biology, 191, 249 (1998), ISSN 0022-5193.

[17] M. Gopalakrishnan, Biophysical Journal, 89, 3686 (2005), ISSN 00063495.

[18] S. Ghosh, M. Gopalakrishnan, and K. Forsten-Williams, Physical Biology, 4, 344 (2008), ISSN 1478-3975.

[19] B. Goldstein and M. Dembo, Biophysical Journal, 68, 1222 (1995), ISSN 0006-3495, PMID: 7787014 PMCID: 1282020 .

[20] B. R. Caré and H. A. Soula, BMC Systems Biology, 5, 48 (2011), ISSN 1752-0509, PMID: 21453460.

[21] B. R. Caré and H. A. Soula, in Information Processing in Cells and Tissues, Vol. 7223 (Springer Berlin Heidelberg, Berlin, Heidelberg, 2012) pp. 50-61, ISBN 978-3642-28791-6, 978-3-642-28792-3.

[22] A. Mugler, A. G. Bailey, K. Takahashi, and P. Rein ten Wolde, Biophysical Journal, 102, 1069 (2012), ISSN 0006-3495.

[23] D. A. Lauffenburger and J. Linderman, Receptors: Models for Binding, Trafficking, and Signaling (Oxford University Press, USA, 1996) ISBN 0195106636.

[24] D. T. Gillespie, Annual Review of Physical Chemistry, 58, 35 (2007), ISSN 0066-426X.

[25] S. Wanant and M. J. Quon, Journal of Theoretical Biology, 205, 355 (2000), ISSN 0022-5193, PMID: 10882558.
[26] A. R. Sedaghat, A. Sherman, and M. J. Quon, American Journal of Physiology - Endocrinology And Metabolism, 283, E1084 (2002).

[27] J. Murray, Mathematical biology: I. An introduction (Springer, 2005).

[28] R. Kopelman, Science, 241, 1620 (1988), ISSN 00368075, 1095-9203.

[29] H. Berry, Biophysical journal, 83, 1891-1901 (2002), ISSN 0006-3495.

[30] L. Boulianne, S. A. Assaad, M. Dumontier, and W. J. Gross, BMC Systems Biology, 2, 66 (2008), ISSN 17520509.

[31] S. R. Post, R. Hilal-Dandan, K. Urasawa, L. L. Brunton, and P. A. Insel, Biochemical Journal, 311, 75 (1995).

[32] D. W. Banner, A. D'Arcy, W. Janes, R. Gentz, H. J. Schoenfeld, C. Broger, H. Loetscher, and W. Lesslauer, Cell, 73, 431 (1993), ISSN 0092-8674, PMID: 8387891.

[33] K. Parang, J. H. Till, A. J. Ablooglu, R. A. Kohanski, S. R. Hubbard, and P. A. Cole, Nature structural biology, 8, 37 (2001), ISSN 1072-8368, PMID: 11135668.

[34] A. Pralle, P. Keller, E.-L. Florin, K. Simons, and J. K. H. Hörber, The Journal of Cell Biology, 148, 997 (2000), ISSN 0021-9525, 1540-8140.

[35] A. Nohe, E. Keating, M. Fivaz, F. G. van der Goot, and N. O. Petersen, Nanomedicine: nanotechnology, biology, and medicine, 2, 1 (2006), ISSN 1549-9642, PMID: 17292110.

[36] D. Shoup and A. Szabo, Biophysical Journal, 40, 33 (1982), ISSN 0006-3495, PMID: 7139033 PMCID: PMC1328970.

[37] R. Lammers, E. Van Obberghen, R. Ballotti, J. Schlessinger, and A. Ullrich, Journal of Biological Chemistry, 265, 16886 (1990).

[38] G. R. Hayes, L. D. Lydon, and D. H. Lockwood, Diabetes, 40, 300 (1991), ISSN 0012-1797, PMID: 1991577.

[39] I. Ji, C. Lee, Y. Song, P. M. Conn, and T. H. Ji, Molecular endocrinology (Baltimore, Md.), 16, 1299 (2002), ISSN 0888-8809, PMID: 12040016.

[40] C. Monnier, H. Tu, E. Bourrier, C. Vol, L. Lamarque, E. Trinquet, J.-P. Pin, and P. Rondard, The EMBO journal, 30, 32 (2011), ISSN 1460-2075, PMID: 21063387.

[41] O. Thoumine, E. Saint-Michel, C. Dequidt, J. Falk, R. Rudge, T. Galli, C. Faivre-Sarrailh, and D. Choquet, Biophysical Journal, 89, L40 (2005), ISSN 00063495.

[42] T. Duke and I. Graham, Progress in Biophysics and Molecular Biology, 100, 18 (2009), ISSN 1873-1732, PMID: 19747931.

[43] H. A. Soula, A. Coulon, and G. Beslon, BMC biophysics, 5, 6 (2012), ISSN 2046-1682, PMID: 22546236.

[44] B. R. Caré and H. A. Soula, in 4th International Conference on Bioinformatics and Computational Biology (BICoB) (2012) ISBN 9781618397461.

[45] Taufiq-Ur-Rahman, A. Skupin, M. Falcke, and C. W. Taylor, Nature, 458, 655 (2009), ISSN 0028-0836, PMID: 19348050 PMCID: 2702691. 